\title{
Real-Time Cubesat Thermal Simulation using Artificial Neural Networks
}

\author{
José D. Reis Junior $^{a 1}$, Ana M. Ambrosio ${ }^{a}$, and Fabiano L. de Sousa ${ }^{a}$ \\ ${ }^{a}$ National Institute for Space Research, São José dos Campos, SP, Brazil
}

Received on December 12, 2016 / Accepted on June 02, 2017

\begin{abstract}
In space systems engineering, the Operational Simulator (OS) is a computational tool that can be used to test and validate the ground control system, to train the flight control operators, and to support the operation of spacecrafts. In order to accomplish these tasks, the OS must produce data of all the spacecraft subsystems in real-time. Among these subsystems, the thermal control subsystem is one of the most demanding in terms of computational cost. In this work we use Artificial Neural Networks (ANN) to learn the thermal behavior of a simple CubeSat model, generated by a thermal analysis software, and then apply it to reproduce that behavior and to generalize for scenarios not presented during training. The results show that the ANNs can simulate the temperatures of the CubeSat with good fidelity and very low computational cost.
\end{abstract}

Keywords: artificial neural networks, real-time simulation, space systems engineering, CubeSat, thermal control subsystem.

\section{Introduction}

The recent advancements in information technology and the increasing necessity to reduce cost and time has led to a new model of space systems engineering, in which computational modeling and simulation has become essential tools for design, development and operation of such systems. One of the main advantages of modeling and simulation is the reduction in the number of hardware models built during the development of a spacecraft. Other advantages include lower costs, shorter times of development, adaptability to design modifications, no problems with transport or logistics, reusability in successive projects, etc. [1].

Modeling and simulation can be used extensively during all the life cycle of a spacecraft, from the conception and design, to development and operation. In this work, we are interested in a software tool that supports the

\footnotetext{
${ }^{1}$ E-mail Corresponding Author: daniel.reis@inpe.br
} 
operations phase of satellites, called Operational Simulator (OS). This simulator can be used before the launch of the satellite, to validate the ground control system, to train the ground operators and to test the operation plans, before applying them to the real system in orbit [2-4].

The OS must be capable to be integrated with the ground control system and respond as if the actual satellite is being operated [5]. To meet this requirement, the simulator needs to run in real-time. Given the complexity of space systems, this can be very challenging.

The design of the thermal control subsystem is usually supported by specialized software used for modeling and analysis [6]. In this software, the thermal system is discretized into a network of nodes (a few thousands for a medium satellite) and differential heat equations are integrated to compute the temperatures of these nodes for a specific scenario and at a given time. For this reason, high fidelity thermal simulations are computationally very expensive, which makes it difficult to use directly in an OS. So it is necessary to seek an alternative capable of providing data on the thermal behavior of the spacecraft in real-time with little loss of fidelity compared to the actual system.

In the literature, there are basically two approaches to solve this problem [7-10]. The first one is to carry out an interpolation over a finite set of selected typical scenarios for which the thermal behavior is known. The disadvantage of this method is the uncertainty of the output for nonstandard scenarios. The second method consists in a simplification of the thermal model, reducing the number of nodes and interactions to save processing time in the integration of the differential equations. The drawback is the loss of accuracy, especially for the standard scenarios.

Artificial Neural Networks (ANNs) have been successfully applied for the solution of problems in various fields of engineering [11-13]. Recently, we proposed the use of ANNs as a potential real-time quantitatively high fidelity estimator of the thermal behavior of a satellite in Earth orbit. This approach was utilized to reproduce the thermal behavior of a simple hypothetical nanosatellite [14] and of the Amazonia-1 satellite [15]. Here we return to the nanosatellite model, also called CubeSat [16], to investigate the capability of ANNs to generalize to scenarios not presented during the training process.

\section{Methodology}

The thermal model of the CubeSat was built using AutoCAD ${ }^{\circledR}$ and Thermal Desktop ${ }^{\circledR}$ software. This thermal CAD model, which can be seen in Fig. 1, consists of a square aluminum box of $10 \times 10 \times 10 \mathrm{~cm}$, with three 
printed circuit boards (PCBs; in green), each one containing one dissipative component (in red). The front walls are obscured so that the interior can be seen.

The orbit used in simulations is polar (inclination equal $90^{\circ}$ ) at $500 \mathrm{~km}$ of altitude. This gives a period of $6000 \mathrm{~s}$ or $100 \mathrm{~min}$. The attitude is stabilized in 3-axis with one side always facing the Earth.

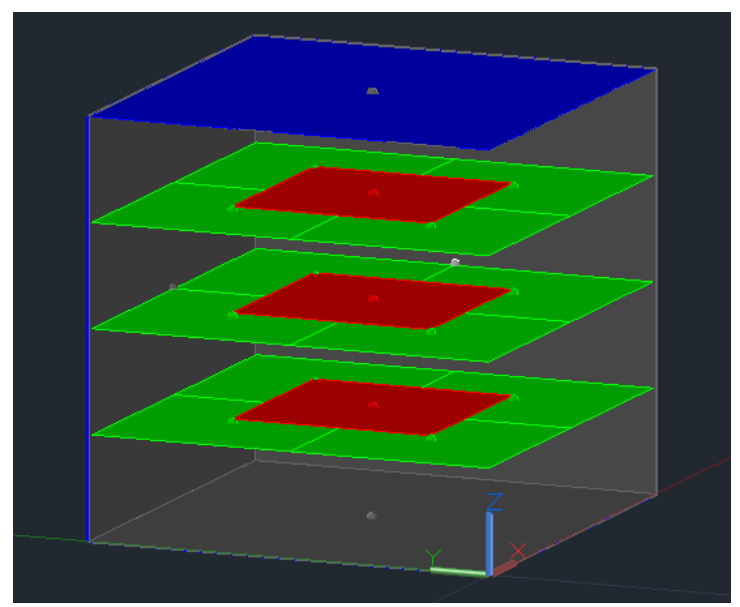

Figure 1 - Thermal CAD model of the CubeSat.

The thermo-physical properties can be seen in Table 1 and the optical properties in Table 2. It was considered that the external surfaces are covered with Solar Cells and the internal surfaces painted with black paint. The dissipative components are composed of Silicon and covered with Graphite Epoxy.

Table 1: Thermo-physical properties

\begin{tabular}{cccc}
\hline Material & $\begin{array}{c}\text { Density } \\
\left(\mathrm{kg} / \mathrm{m}^{3}\right)\end{array}$ & $\begin{array}{c}\text { Thermal Conductivity } \\
\left(\mathrm{W} / \mathrm{m}^{\circ} \mathrm{C}\right)\end{array}$ & $\begin{array}{c}\text { Specific Heat } \\
\left(\mathrm{J} / \mathrm{kg}^{\circ} \mathrm{C}\right)\end{array}$ \\
\hline Aluminum Alloy & 2710 & 168.0 & 963.0 \\
Fiberglass (PCB) & 2440 & 1.1 & 737.0 \\
Silicon & 2320 & 148.8 & 712.0 \\
\hline
\end{tabular}

The thermal modeling is based on a nodal or lumped parameter method. In this method, the satellite is divided in a number of regions, assumed isothermal, which are called nodes. These nodes exchange heat among each 
Table 2: Optical properties

\begin{tabular}{cccc}
\hline Material & Absorptivity $(\alpha)$ & Emissivity $(\varepsilon)$ & $\alpha / \varepsilon$ \\
\hline Fiberglass (PCB) & 0.75 & 0.89 & 0.843 \\
Graphite Epoxy & 0.93 & 0.85 & 1.094 \\
Black Paint & 0.95 & 0.87 & 1.092 \\
Solar Cells & 0.90 & 0.80 & 1.125 \\
\hline
\end{tabular}

other by conduction and radiation and with outer space by radiation. Also, they can receive heat loads from external sources or from electronic components. The temperature of each node is the result of these interactions.

The thermal software package SINDA/FLUINT (SINDA), which is a tool for heat transfer design and fluid flow modeling of complex systems, was applied to calculate the temperatures of the satellite in various scenarios. The first one is an operational scenario with all the components working normally and space environment parameters at its maximum values (hot case). In the second one, the components are in standby and the space environment parameters at its minimum values (cold case). The other scenarios consist in variations of each parameter individually alternating from its maximum, minimum and medium values while maintaining all other variables in its maximum or minimum values. This approach was employed in order to the ANN learn the influence of each parameter in the thermal behavior of the CubeSat. We also simulated two additional arbitrary scenarios, A and B, to test the generalization capability of the ANN. The simulated scenarios can be seen in Table 3.

First, the steady state was calculated and then the transient temperatures were stabilized for 10 orbits. Afterwards, the ANN was trained with two data sets. The first one consists of the last orbit from the scenarios 1 to 14, and the second comprise the last 5 orbits from the scenarios 1 to 27 . The thermal model contains a total of 21 nodes, but only the data of 9 nodes were used for training. These 9 nodes relate to the 6 external surfaces and the 3 internal components. The remaining nodes (PCB's nodes) are important in the computation of the temperature distribution in the satellite, but they are not required in the OS, since the satellite telemetry usually does not contain such information.

To perform training, it was utilized a classical Multilayer Perceptron ANN with supervised learning [17]. The structure of the network consists of 7 elements in the input layer; two hidden layers, with 30 to 50 neurons each; and 9 neurons in the output layer. The elements in the first layer 
Table 3: Simulated scenarios

\begin{tabular}{cccccccc}
\hline$\#$ & Scenario & $\begin{array}{c}\text { Comp.1 } \\
(\mathrm{W})\end{array}$ & $\begin{array}{c}\text { Comp.2 } \\
(\mathrm{W})\end{array}$ & $\begin{array}{c}\text { Comp.3 } \\
(\mathrm{W})\end{array}$ & $\begin{array}{c}\text { Solar } \\
\left(\mathrm{W} / \mathrm{m}^{2}\right)\end{array}$ & $\begin{array}{c}\text { Albedo } \\
\left(\mathrm{W} / \mathrm{m}^{2}\right)\end{array}$ & $\begin{array}{c}\text { Earth } \\
\left(\mathrm{W} / \mathrm{m}^{2}\right)\end{array}$ \\
\hline 1 & Hot & 0.80 & 0.40 & 0.60 & 1418 & 595.56 & 233 \\
2 & Cold & 0.20 & 0.10 & 0.15 & 1326 & 450.84 & 208 \\
3 & MaxCp1 & 0.80 & 0.10 & 0.15 & 1326 & 450.84 & 208 \\
4 & MaxCp2 & 0.20 & 0.40 & 0.15 & 1326 & 450.84 & 208 \\
5 & MaxCp3 & 0.20 & 0.10 & 0.60 & 1326 & 450.84 & 208 \\
6 & MaxSol & 0.20 & 0.10 & 0.15 & 1418 & 450.84 & 208 \\
7 & MaxAlb & 0.20 & 0.10 & 0.15 & 1326 & 595.56 & 208 \\
8 & MaxER & 0.20 & 0.10 & 0.15 & 1326 & 450.84 & 233 \\
9 & MinCp1 & 0.20 & 0.40 & 0.60 & 1418 & 595.56 & 233 \\
10 & MinCp2 & 0.80 & 0.10 & 0.60 & 1418 & 595.56 & 233 \\
11 & MinCp3 & 0.80 & 0.40 & 0.15 & 1418 & 595.56 & 233 \\
12 & MinSol & 0.80 & 0.40 & 0.60 & 1326 & 595.56 & 233 \\
13 & MinAlb & 0.80 & 0.40 & 0.60 & 1418 & 450.84 & 233 \\
14 & MinER & 0.80 & 0.40 & 0.60 & 1418 & 595.56 & 208 \\
15 & Medium & 0.50 & 0.25 & 0.375 & 1372 & 523.20 & 220.5 \\
16 & MedCp1a & 0.50 & 0.40 & 0.60 & 1418 & 595.56 & 233 \\
17 & MedCp1b & 0.50 & 0.10 & 0.15 & 1326 & 450.84 & 208 \\
18 & MedCp2a & 0.80 & 0.25 & 0.60 & 1418 & 595.56 & 233 \\
19 & MedCp2b & 0.20 & 0.25 & 0.15 & 1326 & 450.84 & 208 \\
20 & MedCp3a & 0.80 & 0.40 & 0.375 & 1418 & 595.56 & 233 \\
21 & MedCp3b & 0.20 & 0.10 & 0.375 & 1326 & 450.84 & 208 \\
22 & MedSola & 0.80 & 0.40 & 0.60 & 1372 & 595.56 & 233 \\
23 & MedSolb & 0.20 & 0.10 & 0.15 & 1372 & 450.84 & 208 \\
24 & MedAlba & 0.80 & 0.40 & 0.60 & 1418 & 523.20 & 233 \\
25 & MedAlbb & 0.20 & 0.10 & 0.15 & 1326 & 523.20 & 208 \\
26 & MedERa & 0.80 & 0.40 & 0.60 & 1418 & 595.56 & 220.5 \\
27 & MedERb & 0.20 & 0.10 & 0.15 & 1326 & 450.84 & 220.5 \\
28 & A & 0.63 & 0.12 & 0.48 & 1345 & 551.45 & 229 \\
29 & B & 0.26 & 0.31 & 0.19 & 1398 & 503.28 & 214 \\
\hline & & & & & & & \\
\hline
\end{tabular}

refer to time, the power of the 3 components, Solar Radiation, Albedo, and Earth Radiation. The main parameters used for training were learning rate of 0.01 ; momentum constant of 0.5 ; error tolerance of 0.0001 ; and, in case of non-convergence, the execution was interrupted after $10^{6}$ epochs (complete training iterations). After successful training, the ANN was used to build temperature curves, based on the knowledge acquired. 
In addition to the data provided by the thermal analysis software, the same procedure could be applied using the data from the thermal tests or from the telemetry of the spacecraft after launch.

\section{Results and Discussion}

First, we show the results for the first data set (last orbit). Fig. 2 contains the comparison between the temperature curves generated by the SINDA software (in red) and the ANN (in blue), for two components, in four different scenarios: Hot, Cold, A, and B. The curves are shown as continuous lines and one of the components was omitted for better visualization.
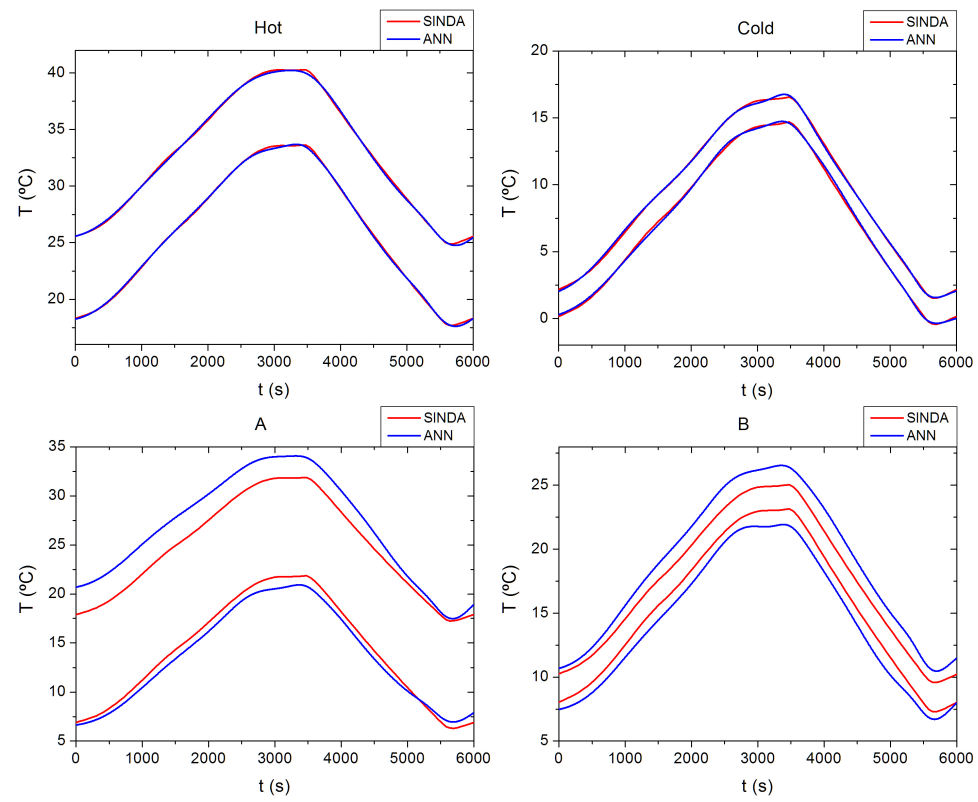

Figure 2 - Comparison of ANN and SINDA curves for the first data set.

For the Hot and Cold cases the curves generated by the ANN show good agreement with the ones produced with SINDA. As for the scenarios $\mathrm{A}$ and $\mathrm{B}$, there is a gap between the data from the two sources. The output generated for the cases $\mathrm{A}$ and $\mathrm{B}$ denote the generalization capability of the ANN, since these scenarios were not used in the training process. The quantitative comparison is listed in Tab. 4. The error for the Hot and Cold cases is less than $1{ }^{\circ} \mathrm{C}$. On the other hand, the max error for the cases $\mathrm{A}$ and $\mathrm{B}$ are respectively $3.04{ }^{\circ} \mathrm{C}$ and $1.79{ }^{\circ} \mathrm{C}$. In thermal control of space systems engineering for generic components an error smaller than $5^{\circ} \mathrm{C}$ is acceptable, so we consider this a very good result. 
Table 4: ANN and SINDA comparison for the first data set

\begin{tabular}{cccc}
\hline Scenario & $\begin{array}{c}\text { Mean Error } \\
\left({ }^{\circ} \mathrm{C}\right)\end{array}$ & $\begin{array}{c}\text { Standard Deviation } \\
\left({ }^{\circ} \mathrm{C}\right)\end{array}$ & $\begin{array}{c}\text { Max Error } \\
\left({ }^{\circ} \mathrm{C}\right)\end{array}$ \\
\hline Hot & 0.11 & 0.08 & 0.70 \\
Cold & 0.11 & 0.07 & 0.59 \\
A & 1.07 & 0.29 & 3.04 \\
B & 0.52 & 0.21 & 1.79 \\
\hline
\end{tabular}

The curves for the second data set (five orbits) are plotted in Fig. 3.
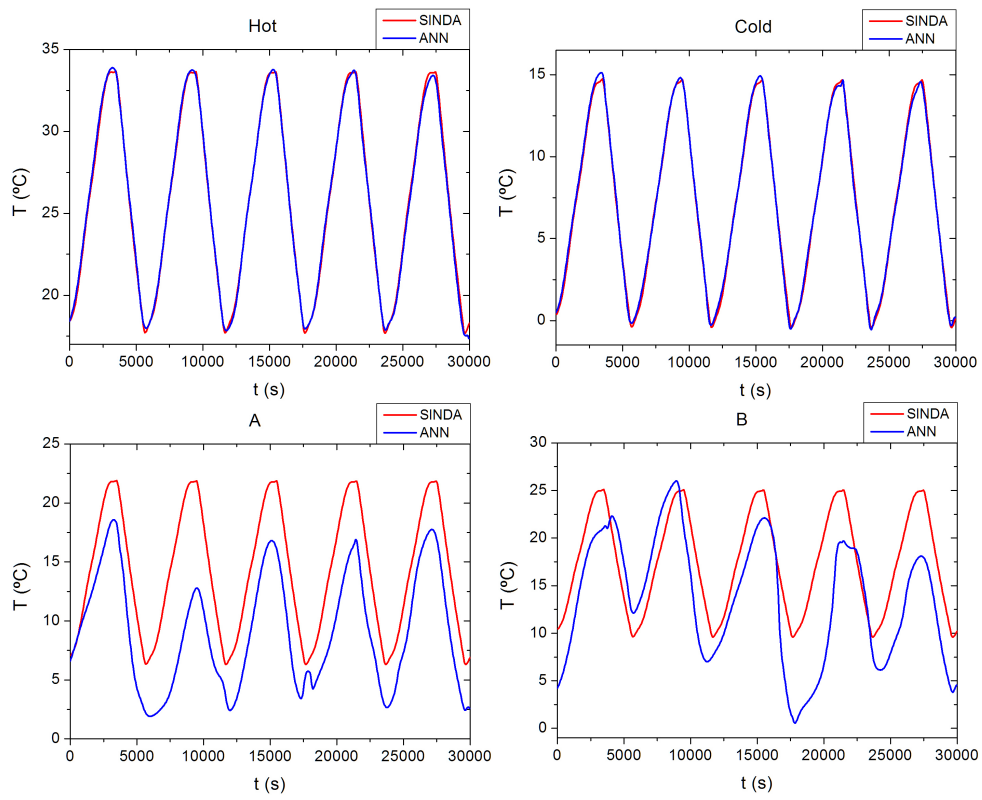

Figure 3 - Comparison of ANN and SINDA curves for the second data set.

This time, the figures contain the data of just one component for better visualization. Again, for the Hot and Cold scenarios the curves generated by the ANN present good agreement with the ones produced with SINDA. However, in the cases A and B the ANN result show a greater difference from the curves of SINDA. In our tests, we observed that if we varied the value of one or two input variables, while keeping the others in values used for training, the resulting curves presented the expected behavior, which are five regular oscillations. Otherwise, if we altered three or more variables simultaneously, the curves diverged from the expected behavior, as shown for the A and B scenarios in Fig. 3. 
Table 5 contains the calculated error for the second data set. As for the first data set, the errors for the Hot and Cold scenarios are very low. For the $\mathrm{A}$ and $\mathrm{B}$ cases, the mean error is $3.59{ }^{\circ} \mathrm{C}$ and $5.59{ }^{\circ} \mathrm{C}$, respectively. What considered alone would be acceptable. Nevertheless, the maximum error observed is greater than $20{ }^{\circ} \mathrm{C}$ for some specific points. This is more than the acceptable limit mentioned above and we could not find better results for the range of parameters tested.

\begin{tabular}{cccc}
\multicolumn{2}{c}{ Table 5: ANN and SINDA comparison for the second data set } \\
\hline Scenario & $\begin{array}{c}\text { Mean Error } \\
\left({ }^{\circ} \mathrm{C}\right)\end{array}$ & $\begin{array}{c}\text { Standard Deviation } \\
\left({ }^{\circ} \mathrm{C}\right)\end{array}$ & $\begin{array}{c}\text { Max Error } \\
\left({ }^{\circ} \mathrm{C}\right)\end{array}$ \\
\hline Hot & 0.16 & 0.12 & 1.16 \\
Cold & 0.15 & 0.13 & 1.06 \\
A & 3.59 & 3.14 & 20.90 \\
B & 5.59 & 4.47 & 21.76 \\
\hline
\end{tabular}

One possible reason for the difference from the results of the two data sets is the greater number of information the ANN has to learn in the second one. Besides that, we consider that the results for the second data set are good results, because the mean error is not very high and the scenarios A and $\mathrm{B}$ are actually extreme cases where all the variables were modified from the trained values simultaneously. Additionally, for the Operational Simulator we will only need one orbit, due to the cyclic behavior of the temperatures for a given set of parameters as a function of the orbit period.

\section{Conclusion}

The results for the first data set, containing just one orbit, showed very low error in the data produced by the ANN, not only for the scenarios used for training but also for arbitrary ones. In this case, the ANN provided good generalization, i.e. generated reasonable outputs, for data not used in training.

The results for the second data set (containing five orbits) also showed very low error for the curves produced by the ANN in comparison with the training set. On the other hand, the ANN had more difficulty in generalizing for arbitrary configurations of the input variables, especially when all the variables were modified at the same time.

In summary, the MLP neural network is very efficient in learning from data and reproducing this data after training. However, the generalization ability of this type of ANN is very dependent on the parameters of the 
network and on the complexity of the data set.

Considering this fact, our current objective is to further analyze the influence of each parameter in the generalization capability of the ANN. Afterwards, we intend to increase the scale of the problem, including larger satellites, and apply more modern methods of ANNs such as Deep Learning [18].

\section{References}

[1] Hendricks, R. and Eickhoff, J., The Significant Role of Simulation in Satellite Development and Verification, Aerospace Science and Technology, vol. 9, 273283, 2005.

[2] Ambrosio, A.M., Cardoso, P. E., Orlando, V., and Bianchi-Neto, J., Brazilian Satellite Simulators: Previous Solutions Trade-off and New Perspectives for the CBERS Program, Proceedings of the $8^{\text {th }}$ Conference on Space Operations (SPACEOPS), 2006.

[3] Eickhoff, J., Simulating Spacecraft Systems, Springer-Verlag Berlin Heidelberg, 2009.

[4] Zemerick, S.A., Morris, J.R., and Bailey, B.T., NASA Operational Simulator (NOS) for V\&V of Complex Systems, Proceedings of SPIE Volume 8752: Modeling and Simulation for Defense Systems and Applications VII, 2013.

[5] Reggestad, V., Pecchioli, M., and Merri, M., Virtual Reality for Real Operations, ESA Bulletin, n. 148, p. 43, 2011.

[6] Gilmore, D.G., Spacecraft Thermal Control Handbook, The Aerospace Press, vol. 1, $2^{\text {nd }}$ edition, 2002.

[7] Perpiñán, M.A.C., The Modelling of the Thermal Subsystem in Spacecraft Real Time Simulators, Proceedings of the $3^{\text {rd }}$ Workshop on Simulators for European Space Programmes, ESA/ESTEC, pp. 69-78, 1994.

[8] Kang, J.Y., Kim, J.M., and Chung, S.J., Design and Development of an Advanced Real-Time Satellite Simulator, ETRI Journal, vol. 17, n. 3, 1995. 
[9] Martínez-Heras, J.A. and Donati, A., Artificial Neural Networks in Support of Spacecraft Thermal Behaviour Modeling, IEEE Aerospace Conference Proceedings, 2004.

[10] Raif, M., Walter, U., and Bouwmeester, J., Dynamic System Simulation of Small Satellite Projects, International Astronautical Congress Proceedings, IAC-09.D1.6.8, 2009.

[11] Yang, K.T., Artificial Neural Networks (ANNs): A New Paradigm for Thermal Science and Engineering, Journal of Heat TransferTransactions of the ASME, vol. 130, $9^{\text {th }}$ edition, 093001, 2008.

[12] Pei, J.S. and Mai E.C., Constructing Multilayer Feedforward Neural Networks to Approximate Nonlinear Functions in Mechanical Engineering Applications, Journal of Applied Mechanics-Transactions of the ASME, vol. 75, $6^{\text {th }}$ edition, 061002, 2008.

[13] Nguyen, N. et al., Repetitively Enhanced Neural Networks Method for Complex Engineering Design Optimisation Problems, Aeronautical Journal, vol. 119, ed. 1220, pp. 1253-1270, 2015.

[14] Reis Junior, J.D., Ambrosio, A.M., and Sousa, F.L., Towards Spacecraft Real-Time Thermal Simulation with Artificial Neural Networks, Proceedings of the 23rd ABCM International Congress of Mechanical Engineering, 0316-21880, 2015.

[15] Reis Junior, J.D., Ambrosio, A.M., and Sousa, F.L., Reproducing Amazonia-1 Satellite Thermal Behavior with Artificial Neural Networks, Proceedings of the $7^{\circ}$ Workshop em Engenharia e Tecnologia Espaciais, São José dos Campos, Brazil, 2016.

[16] Woellert, K., Ehrenfreund, P., Ricco, A.J., and Hertzfeld, H., Cubesats: Cost-effective science and technology platforms for emerging and developing nations, Advances in Space Research, vol. 47, pp. 663-684, 2011.

[17] Haykin, S., Neural Networks and Learning Machines, Pearson Education, New Jersey, $3^{\text {rd }}$ edition, 2009.

[18] LeCun, Y., Bengio, Y, Hinton, G., Deep Learning, Nature, vol. 521, ed. 7553, pp. 436-444, 2015. 